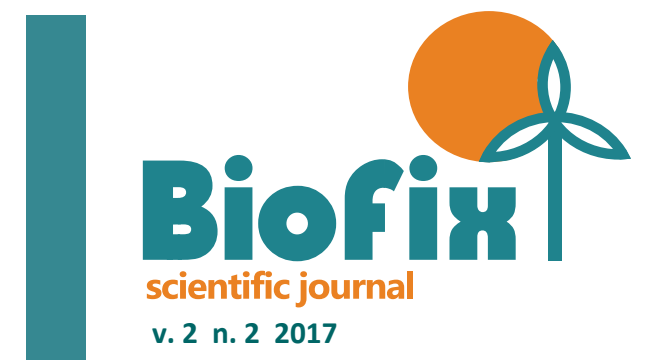

v. 2 n. 22017

Recebido em 31/08/2017

Aceito em 05/10/2017

Publicado em 10/10/2017

DOI: dx.doi.org/10.5380/biofix.v2i2.54954

D.:

\section{MANUTENÇÃO PREVENTIVA COMO DETERMINANTE PARA REDUÇÃO DE CUSTOS DE MANUTENÇÃO DE UM FELLER BUNCHER}

\author{
PREVENTIVE MAINTENANCE AS A DETERMINANT FOR REDUCTION \\ MAINTENANCE COSTS OF A FELLER BUNCHER
}

\author{
MAINTENANCE COSTS OFAFELLER BUNCHER
}




\section{INTRODUÇÃO}

Equipamentos florestais foram desenvolvidos ao longo do tempo para atender a demanda das empresas em maximizar lucro, de modo a proporcionar aumento na produtividade das operações. Mesmo com adoção de novas tecnologias na engenharia de construção de equipamentos, o setor florestal necessita por dados confiáveis para a escolha do sistema e do equipamento adequado para cada tipo de operação (NASCIMENTO et al., 2011).

Para a operação de corte florestal, muitas empresas utilizam o trator feller buncher, que possui elevada potência nominal, rodado de esteiras ou pneus, nivelamento da cabine e tecnologia embarcada, chegando a produzir em média $115 \mathrm{~m}^{3} \cdot \mathrm{h}^{-1}$ em plantios de baixo volume individual (SIMÕES et al., 2014). Contudo, a produção e a produtividade desse equipamento acabam sofrendo perdas, devido falhas mecânicas e hidráulicas que demandam elevado tempo da operação, diminuindo a disponibilidade mecânica e, por consequência, o tempo produtivo da operação.

A operação de manutenção mecânica é definida como a combinação de todas as atividades administrativas e técnicas que tem por objetivo a manutenção de equipamentos, de instalações e de outros ativos físicos em condições operacionais, mantendo as suas atividades fins (MOUBRAY, 2000; KARDEC et al., 2002; MUCHIRI et al., 2011; MISHRA et al., 2015). Dado o cenário atual, as empresas vêm buscando avaliar todo futuro investimento e, nesse contexto, a manutenção de equipamentos de colheita florestal mostra-se como ponto chave, uma vez que seus custos podem variar de 50 a $60 \%$ do custo total do equipamento (SILVA et al., 2010; LOPES et al., 2014).

Yamashina (2000) comenta que um dos principais modelos de manutenção utilizados é o World Class Maintenance (WCM), que utiliza como base a manutenção preventiva. Dessa forma, o WCM tem por objetivo alcançar a manutenção de classe mundial por meio da liderança de processos, envolvendo as boas práticas de manutenção preventiva.

Apesar da grande quantidade de trabalhos científicos na área de colheita de madeira, existem poucos dados sobre a real influência da manutenção preventiva na operação dos equipamentos florestais. As estimativas de disponibilidade mecânica e de custo baseadas em dados fornecidos pelos fabricantes ou obtidas em trabalhos realizados em outros países mostram-se bastante frágeis, indicando, portanto, a necessidade da realização de estudos para cada condição específica (PEREIRA et al.,
2015).

Diante disso, este trabalho objetivou avaliar a atividade de manutenção mecânica de um feller buncher usando o modelo de manutenção WCM, que emprega a manutenção preventiva como premissa, visando a redução dos custos de produção e o melhor aproveitamento dos recursos disponíveis.

\section{MATERIAL E MÉTODOS}

A pesquisa foi realizada em uma empresa florestal localizada no estado do Paraná, Brasil. Segundo a classificação climática de Köppen (1948), o clima da região é caracterizado como transição entre $\mathrm{Cfa}$ e $\mathrm{Cfb}$, com chuvas regulares e precipitação média anual entre 1.478 a 1.700 $\mathrm{mm}$.

A área de estudo correspondeu a plantios de Eucalyptus grandis e Pinus taeda com 7 e 17 anos, respectivamente. $\mathrm{O}$ volume individual dos povoamentos variou entre $0,36 \mathrm{~m}^{3}$ e 0,42 $\mathrm{m}^{3}$. O relevo da área estudada é caracterizado como suavemente ondulado, sendo que a operação de corte foi realizada sob regime de corte raso.

O objeto de estudo foi um feller buncher com potência nominal de $300 \mathrm{hp} / 224 \mathrm{Kw}$ equipado com motor Cummins QSL9 Tier III (diesel) com peso operacional de 38 toneladas e horímetro de 4.977 horas (Figura 1).

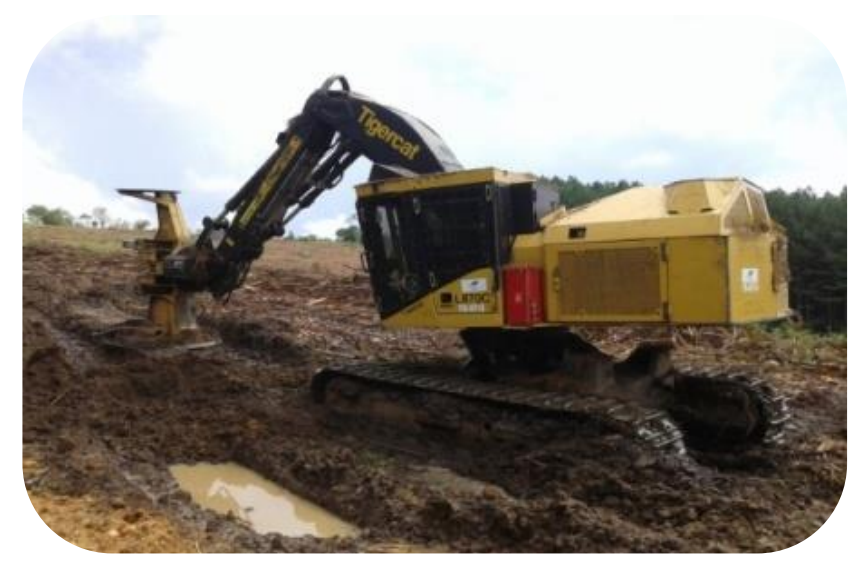

Figura 1. Feller buncher utilizado para o corte florestal de povoamentos de pinus e eucalipto.

As manutenções preventivas foram realizadas em um ciclo contínuo de oito semanas, onde em cada semana foram inspecionados, revisados, substituídos e reparados uma série de componentes dos sistemas hidráulico, elétrico, mecânico e eletrônico.

Outro fator de diferenciação do modelo de manutenção empregado na pesquisa é que o mesmo contou com almoxarifado de campo equipado com peças que apresentaram maior grau de falhas, treinamento de 
mecânicos e operadores para a atividade de manutenção mecânica.

Os dados foram coletados a partir de diários de bordo do equipamento obtidos junto a empresa por um período de 18 meses, sendo dividido em três estágios de igual duração, implantação, amadurecimento e estabilização das atividades.

Para verificar a operação de manutenção, foram calculados os índices de disponibilidade mecânica e consumo de óleo hidráulico, sendo, posteriormente, calculado o custo de manutenção da máquina.

A disponibilidade mecânica, definida como o tempo em que o equipamento está disponível mecanicamente para realizar o trabalho, foi obtida pela Equação 1 (SANTOS et al., 2013; LOPES et al., 2016; GUEDES et al., 2017).

$$
\mathrm{DM}=\frac{\mathrm{HT}-\mathrm{HM}}{\mathrm{HT}} \times 100
$$

Em que: $\mathrm{DM}$ = grau de disponibilidade mecânica (\%); $\mathrm{HT}=$ horas de trabalho programado; e $\mathrm{HM}=$ horas de manutenção.

O consumo médio de óleo hidráulico foi calculado a partir de uma Equação 2, adaptada de Nascimento et al. (2011) e Simões et al. (2014).

$$
\mathrm{COH}=\frac{\mathrm{LOH}}{\mathrm{H}}
$$

Em que: $\mathrm{COH}=$ consumo médio de óleo hidráulico $\left({\mathrm{L} . h^{-1}}^{-1}\right) ; \mathrm{LOH}=$ quantidade de óleo hidráulico consumido no mês (L); e H = quantidade de horas trabalhadas no mês.

O custo de manutenção do feller buncher foi realizado com base na determinação dos custos de pessoal, materiais, serviços externos e apoio por hora efetivamente trabalhada, sendo utilizados valores observados e estimados.

O custo de pessoal expresso na Equação 3, incluiu as despesas com salários, encargos sociais e benefícios fornecidos pela empresa, como participação nos lucros, despesas com os treinamentos e reciclagens da equipe de manutenção.

$$
C P=\frac{S L+E N+B N+T R}{H T}
$$

Em que: $\mathrm{CP}=$ custo de pessoal $\left(\mathrm{R} \$ \mathrm{~h}^{-1}\right) ; \mathrm{SL}=$ salários $(\mathrm{R}) ; \mathrm{EN}=$ encargos $(\mathrm{R} \$) ; \mathrm{BN}=$ benefícios $(\mathrm{R} \$) ; \mathrm{TR}=$ treinamentos e reciclagens $(R \$)$; e $H T$ = quantidade de horas efetivamente trabalhadas no mês.

$\mathrm{O}$ custo de materiais referiu-se à reposição de peças, consumo de água, capital imobilizado, gestão dos almoxarifados e o setor de compras, como mostrado na Equação 4.

$$
\mathrm{CMT}=\frac{\mathrm{MT}}{\mathrm{HT}}
$$

Em que: $\mathrm{CMT}=$ custo de materiais $\left(\mathrm{R} \$ \mathrm{~h}^{-1}\right) ; \mathrm{MT}=$ consumo de materiais (R\$); e HT = quantidade de horas trabalhadas no mês.

O custo de serviços externos obtido pela Equação 5 referiu-se aos valores mensais dos contratos com empresas terceirizadas para execução de serviços, tais como: lavagem dos equipamentos, soldas e análise de óleos.

$$
\mathrm{CSE}=\frac{\mathrm{CT}}{\mathrm{HT}}
$$

Em que: $C S E$ = custo de serviços externos $\left(R \$ \cdot h^{-1}\right)$; $\mathrm{CT}=$ contratos de terceiros (R\$); e HT = quantidade de horas trabalhadas no mês.

O custo de apoio referiu-se aos insumos utilizados pelos equipamentos que compõem a infraestrutura de manutenção, tais como: veículos de apoio, caminhão comboio, veículo utilizado para realização de soldas, almoxarifado, etc., conforme mostrado na Equação 6.

$$
\mathrm{CA}=\frac{\mathrm{Cl}}{\mathrm{HT}}
$$

Em que: $\mathrm{CA}=$ custo de apoio ( $\mathrm{R} \$$ /hora); $\mathrm{Cl}=$ custo dos insumos; e HT = quantidade de horas trabalhadas no mês.

O custo total de manutenção foi obtido pelo somatório dos custos de pessoal, materiais, serviços e apoio, calculado pela Equação 7 .

$$
\mathrm{CM}=\mathrm{CO}+\mathrm{CMT}+\mathrm{CSE}+\mathrm{CA}
$$

Em que: $C M=$ custo de manutenção $\left(R \$\right.$ h $\left.^{-1}\right)$; $\mathrm{CP}=$ custo de pessoal $\left(\mathrm{R} \$ \mathrm{he}^{-1}\right) ; \mathrm{CMT}=$ custo de materiais $\left(R \$ . h e^{-1}\right) ;$ CSE = custo de serviços $\left(R \$ . h e^{-1}\right) ;$ e $C A=$ custo de apoio $\left(R \$ . h e^{-1}\right)$.

Os resultados foram submetidos à análise de variância, sendo as variâncias dos tratamentos avaliadas quanto a sua homogeneidade pelo teste de Bartlett ( $p$-valor $<0,05$ ). As variáveis cujas variâncias mostraram-se homogêneas tiveram os efeitos dos tratamentos testados por meio do teste de $F$ ( $p$-valor <0,05). Quando os resultados revelaram existir diferenças estatisticamente significantes entre médias de tratamentos, as médias foram comparadas pelo teste de Tukey ( $p$-valor $<0,05)$. 


\section{RESULTADOS E DISCUSSÃO}

As atividades de manutenção preventiva aplicadas no equipamento permitiram um sutil aumento da disponibilidade mecânica (Figura 2), contudo, não foi possível detectar diferença significativa entre os estágios pelo teste de Tukey ( $p$-valor $<0,05)$.

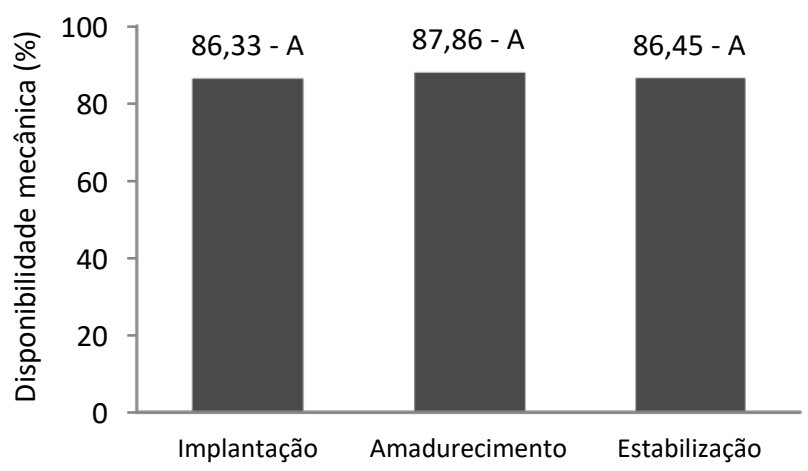

Figura 2. Disponibilidade mecânica do feller buncher nos três estágios avaliados.

Entretanto, as intervenções semanais realizadas na operação e o almoxarifado de campo permitiram maior fluxo da atividade, mesmo quando da necessidade de realização de manutenções corretivas, o tempo de atendimento era menor, com consequente retorno da máquina à atividade em curto espaço de tempo.

A disponibilidade mecânica do feller buncher foram inferiores aos valores obtidos por Simões et al. (2014) e Pereira et al. (2015), os quais obtiveram valores superiores a $90 \%$. Entretanto, tais resultados foram obtidos em estudos realizados em curto período de tempo, enquanto neste estudo obteve-se dados por um período de 18 meses, permitindo a obtenção de resultados concretos e confiáveis.

Os resultados de consumo de óleo hidráulico nos três estágios avaliados apresentaram redução abrupta no consumo de óleo hidráulico a partir do estágio de amadurecimento (Figura 3), apresentando ainda diferença significativa pelo teste de Tukey ( $p$-valor $<0,05$ ).

A realização de manutenções preventivas é um dos fatores que explicam a redução abrupta de $63 \%$ no consumo de óleo hidráulico, o que possibilitou a substituição de reparos e mangueiras desgastados, além do reaperto de peças que apresentavam folgas visando evitar o vazamento de óleo hidráulico e o contato de peças que provoca o desgaste prematuro.

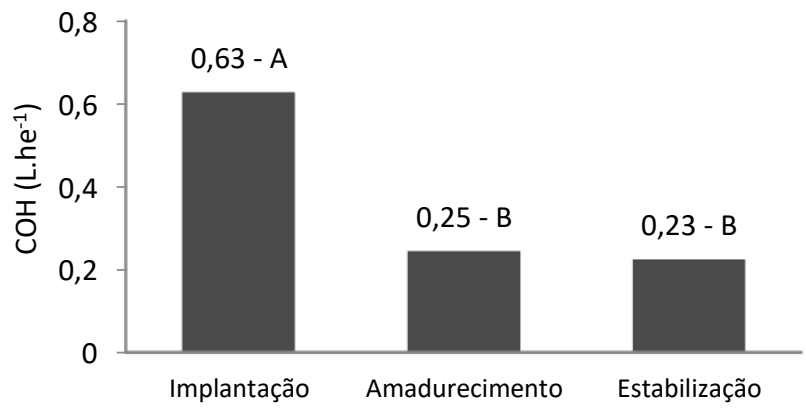

Figura 3. Consumo de óleo hidráulico do feller buncher nos três estágios avaliados.

Observando a Figura 4, pode-se constatar que, com a realização das manutenções preventivas, foi possível reduzir o custo de manutenção do feller buncher em 21\%, sendo que tais resultados apresentaram diferença estatística pelo teste de Tukey ( $p$-valor $<0,05)$. Além disso, é observado aumento no custo de manutenção no estágio de amadurecimento, explicado pelo investimento feito com treinamentos de mecânicos e aquisição de peças e ferramentas.

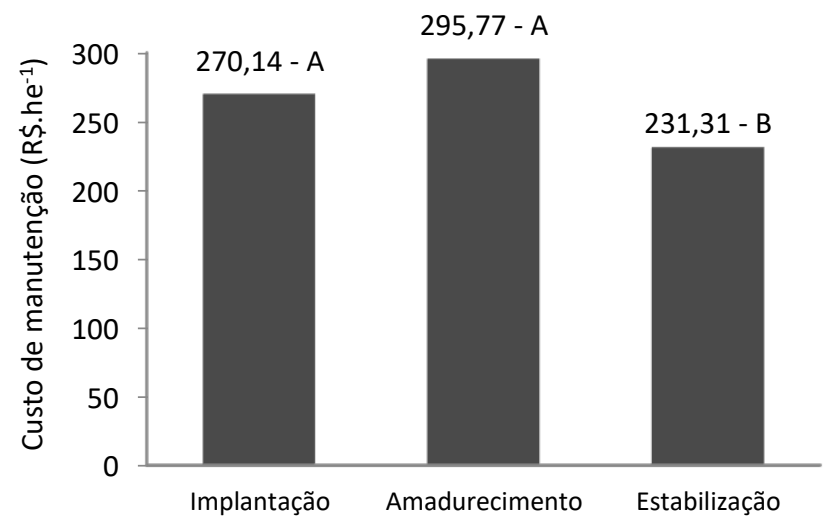

Figura 4. Custo de manutenção do feller buncher nos três estágios avaliados.

Estudos têm apontado que o tipo de manutenção empregada nos equipamentos de colheita de madeira é muito sensível às condições operacionais de cada empresa, como nível de treinamento de mecânicos e de operadores, logística de suprimento de peças e combustíveis, tamanho da estrutura de manutenção no campo, entre outros (MALINOVSKI et al., 2006; FIEDLER et al., 2008; LOPES et al., 2008; FERNANDES et al., 2013; SCHETTINO et al., 2015). Esses, em conjunto ou isoladamente, acabam neutralizando as reduções de custos e os aumentos de disponibilidade mecânica dos equipamentos de colheita. 


\section{CONCLUSÕES}

A utilização do modelo de manutenção WCM proporciona aumento sutil da disponibilidade mecânica do feller buncher, indicando a possibilidade de aumento da produção da operação de corte florestal.

Há redução abrupta no consumo médio de óleo hidráulico, justificado pela realização de reaperto de terminais e substituição de mangueiras e reparos com desgaste, evitando assim o vazamento de óleo durante a colheita de madeira.

O modelo de manutenção WCM proporciona redução do custo de manutenção do feller buncher, evidenciando que a aplicação de práticas de manutenção preventiva oferece maior regularidade na produtividade das operações de corte florestal.

\section{REFERÊNCIAS}

FERNANDES, H. C.; BURLA, E. R.; LEITE, E. S.; MINETTE, L. J. Avaliação técnica e econômica de um "Harvester" em diferentes condições de terreno e produtividade da floresta. Scientia Forestalis, v. 41, n. 97, p. 145-151, 2013.

FIEDLER, N. C.; ROCHA, E. B.; LOPES, E. S. Análise da produtividade de um sistema de colheita de árvores inteiras no Norte do Estado de Goiás. Floresta, v. 38, n. 4, p. 577-586, 2008.

GUedes, L. G.; AMARAL, E. J.; LeITE, E. S.; FeRnANDES, H. C.; SANT'ANNA, C. M. Avaliação do desempenho e custos de dois sistemas de cabos aéreos na extração de madeira de eucalipto. Ciência Florestal, v. 27, n. 2, p. 571-580, 2017.

KARDEC, A.; NASCIF, J.; BARONI, T. Gestão estratégica e técnicas preditivas. Rio de Janeiro: Qualitymark, 2002. 136 p.

KÖPPEN, W. Climatologia: con un estudio de los climas de La tierra. México: Fundo de Cultura Econômica, 1948. 496 p.

LOPES, S. E.; FERNANDES, H. C.; SANTOS, N. T.; RINALDI, P. C. N. Avaliação técnica e econômica de uma garra traçadora operando em diferentes produtividades. Scientia Forestalis, v. 36, n. 79, p. 215-222, 2008.

LOPES, E. S.; OLIVEIRA D.; SAMPIETRO J. A. Influence of wheeles types of a skidder on productivity and cost of the forest harvesting. Floresta, v. 44, n. 1, p. 53-62, 2014.

LOPES, E. S.; DINIZ, C. C. C.; SERPE, E. L.; CABRAL, O.M. J. V. Efeito do sortimento da madeira na produtividade e custo do forwarder no desbaste comercial de Pinus taeda. Scientia Forestalis, v. 44, n. 109, p. 57-66, 2016.

MALINOVSKI, R. A.; MALINOVSKI, R. A.; MALINOVSKI, J. R.; YAMAJI, F. M. Análise das variáveis de influência na produtividade das máquinas de colheita de madeira em função das características físicas do terreno, do povoamento e do planejamento operacional florestal. Floresta, v. 36, n. 2, p. $169-$ 182, 2006.
MISHRA, R. P.; KODALI, R. B.; GUPTA, G.; MUNDRA, N. Development of a framework for implementation of world-class maintenance systems using interpretative structural modeling approach. Procedia CIRP, v. 26, p. 424-429, 2015.

MOUBRAY, J. Manutenção Centrada em Confiabilidade. 2 ed. Lutterworth: Aladon Ltd., 2000. 256 p.

MUCHIRI, P.; PINTELON, L.; GELDERS, L.; MARTIN. H. Development of maintenance function performance measurement framework and indicators. International Journal of Production Economics, v. 131, n. 1, p. 295-302, 2011.

NASCIMENTO, A. C.; LEITE, A. M. P.; SOARES, T. S.; FREITAS, L. C. Avaliação técnica e econômica da colheita florestal com fellerbuncher. Cerne, v. 17, n. 1, p. 9-15, 2011.

PERREIRA, N. A.; LOPES, E. S.; DIAS, A. N. Análise técnica e de custo do feller buncher e skidder na colheita de madeira em diferentes produtividades do povoamento. Ciência Florestal, v. 25, n. 4, p. 981-989, 2015.

SANTOS, P. H. A.; SOUZA, A. P.; MARZANO, F. L. C.; MINETTE, L. J. Produtividade e custos de extração de madeira de eucalipto com clambunk skidder. Revista Árvore, v. 37, n. 3, p. 511-518, 2013.

SCHETTINO, S.; MINETTE, L. J.; SOUZA, A. P. Correlação entre volumetria de florestas de eucalipto e produtividade e custos de máquinas de colheita de madeira. Revista Árvore, v. 39, n. 5, p. 935-942, 2015.

SILVA, E. M.; MACHADO, C. C.; MINETTE, L. J.; SOUZA, A. P.; FERNANDES, H. C.; SILVA, M. L.; JACOVINE, L. A. Avaliação técnica e econômica do corte mecanizado de Pinus sp. com harvester. Revista Árvore, v. 34, n. 4, p. 745-753, 2010.

SIMÕES, D.; FENNER, P. T.; ESPERANCINI, M. S. T. Produtividade e custos do feller-buncher e processador florestal em povoamento de eucalipto de primeiro corte. Ciência Florestal, v. 20, n. 3, p. 621-630, 2014.

YAMASHINA, $\mathrm{H}$. Challenge to world class manufacturing. International Journal of Quality of Reliability Management, $v$. 12, n. 34, p. 30-31, 2000. 